\title{
Safety and Feasibility of Topical Application of Limonene as a Massage Oil to the Breast
}

\author{
Jessica A. Miller, ${ }^{1, *}$, Patricia A. Thompson" ${ }^{1}$, Iman A. Hakim², Ana Maria Lopez ${ }^{1}$, \\ Cynthia A. Thomson ${ }^{2,3}$, Wade Chew ${ }^{1}$, Chiu-Hsieh Hsu $^{2}$, H.-H. Sherry Chow ${ }^{1}$
}

${ }^{1}$ The University of Arizona Cancer Center, Tucson, USA; ${ }^{2}$ Mel \& Enid Zuckerman College of Public Health, The University of Arizona, Tucson, USA; ${ }^{3}$ Department of Nutritional Sciences, The University of Arizona, Tucson, USA.

Email: jam1@email.arizona.edu

Received July $10^{\text {th }}, 2012$; revised August $12^{\text {th }}, 2012$; accepted August $23^{\text {rd }}, 2012$

\begin{abstract}
Background: Limonene, a major component in citrus oil, has demonstrated anti-cancer effects in preclinical mammary cancer models. However, the effective oral dose translates to a human dose that may not be feasible for chronic dosing. We proposed to evaluate topical application of limonene to the breast as an alternative dosing strategy. Materials and Methods: We conducted a mouse disposition study to determine whether limonene would be bioavailable in the mammary tissue after topical application. SKH-1 mice received topical or oral administration of limonene in the form of orange oil every day for 4 weeks. Plasma and mammary pads were collected $4 \mathrm{hrs}$ after the final dosing. We also conducted an exploratory clinical study to evaluate the safety and feasibility of topically applied limonene in the form of orange oil to the breast. Healthy women were recruited to apply orange oil containing massage oil to their breasts daily for four weeks. Safety and feasibility were assessed by reported adverse events, clinical labs, and usage compliance. Pre and post-intervention nipple aspirate fluid (NAF) and plasma were collected for limonene concentration determination. Results: The mouse disposition study showed that topical and oral orange oil administration resulted in similar mammary tissue disposition of limonene with no clinical signs of toxicity. In the clinical study, the topical application of limonene containing massage oil to the breast was found to be safe with high levels of usage compliance for daily application, although NAF and plasma limonene concentrations were not significantly changed after the massage oil application. Conclusions: Our studies showed that limonene is bioavailable in mammary tissue after topical orange oil application in mice and this novel route of administration to the breast is safe and feasible in healthy women.
\end{abstract}

Keywords: Limonene; Topical Application; Safety

\section{Introduction}

Limonene, a monocyclic monoterpene, is a major component of the essential oils of citrus fruits. It has been associated with diverse biological activities, including antitumor activity, with the most compelling results reported in mammary cancer models. In carcinogen induced rat mammary carcinogenesis models, limonene fed during the initiation or promotion/progression stage inhibits the development of tumors induced by 7,12-dimethylbenz(a)anthracene (DMBA) or tumors induced by $N$-methyl- $N$-nitrosourea (NMU) [1-3]. Dietary feeding of limonene also inhibits the development of ras oncogene-induced mammary carcinomas in rats [4]. Limonene has also been shown to exert chemotherapeutic activity. Oral feeding of limonene results in significant regression of both DMBA- and NMU-induced mammary

"Corresponding author. carcinoma in a dose dependent manner, without any observable systemic toxicity $[2,5]$.

A major limitation for further development of limonene for breast cancer prevention or treatment is that the effective doses determined in animal studies translate to human equivalent doses not feasible for chronic human intake. We proposed to evaluate topical application of limonene to the breast as an alternative dosing strategy because limonene has long been explored as an enhancer for dermal absorption of pharmaceutical drugs by interacting with intercellular stratum corneum lipids to increase diffusibility of drugs through the skin [6,7]. In addition, in vitro studies using isolated human skin have shown that topically applied limonene can penetrate through human epidermis and dermis [8]. Here, we report a mouse tissue disposition study and a clinical safety and tolerability study conducted to evaluate the safety and feasibility of topical limonene administration to the breast. 


\section{Methods}

\subsection{Study Agent}

Limonene-containing essential oils are already in use in aromatherapy massage with no reported clinical toxicity. We have therefore used a similar preparation for both animal and human studies to expedite the conduct of clinical research. Organicorange essential oil (NOW Foods, Bloomingdale, IL, USA) was selected as the source of limonene. The limonene content in the orange oil was quantified by gas chromatography-mass spectrometry (GC-MS) analysis and found to be $93 \%$. Essential oils are typically diluted with carrier or base oil prior to use for massage. We selected the fractionated coconut oil (From Nature with Love, Oxford, CT, USA) as the base oil because it is a light, non-greasy, non-staining, liquid oil. There are claims that it helps to carry therapeutical oils under the skin [9].

\subsection{Mouse Disposition Study}

Pathogen-free hairless mice (female SKH-1 mice, 4-5 weeks of age, $19.5-22.3 \mathrm{~g}$ ) were purchased from Charles River Laboratories (Wilmington, MA, USA). These mice werehoused in temperature and light-controlled rooms and were given water and fed ad libitum. Mice were divided into seven experimental groups $(\mathrm{N}=$ 4 per group); untreated control, topical control receiving only topical application of base oil, oral control receiving only oral administration of base oil, topical application of $10 \%$ orange oil in base oil, topical application of $20 \%$ orange oil in base oil, oral administration of $10 \%$ orange oil in base oil, and oral administration of $20 \%$ orange oil in base oil. For topical application, $80 \mu \mathrm{L}$ of the mixed oil or base oil were applied to the skin directly above the mammary pads. An Elizabethan collar was placed on the mice for $20 \mathrm{~min}$ after the topical application to prevent the mice from licking the topically applied oil. For oral administration, $80 \mu \mathrm{L}$ of the mixed oil or base oil were administered via oral gavage. The dose of limonene administered was determined to be 6.0 and $12 \mathrm{mg}$ for the $10 \%$ and $20 \%$ mixed oil, respectively. The administration was carried out daily for four weeks. At the end of the four-week administration, mice from each treatment group were sacrificed 4 hours after the last application/dosing. Plasma and left mammary pads were collected for limonene concentration determination. The fourth right mammary fat pad was collected for wholemount histological evaluation [10]. The experimental protocols for the animal experiments were approved by the Institutional Animal Care and Use Committee at the University of Arizona. Studies were performed according to these ethical guidelines.

\subsection{Human Safety and Tolerability Study}

We recruited women who were 18 - 65 years of age, had good performance status, had normal organ and marrow function, were willing to use adequate contraception, were willing to avoid citrus products throughout the study, and had both breasts intact for study participation. Women were excluded if they were pregnant or breast feeding, had invasive cancers within the past 5 years, participated in another clinical intervention trial within the past 3 months, had uncontrolled severe metabolic disorders or other serious acute or chronic diseases, were unable to produce nipple aspirate fluid, had known allergic or sensitive reactions to skin care products, citrus or coconut oil, or had ongoing skin disorders such as eczema and psoriasis. The study was approved by the University of Arizona Human Subjects Committee and written consent was obtained from all participants.

At the initial clinic visit, participants had a blood sample collected for complete blood count (CBC) with differential and a comprehensive blood chemistry panel. A urine pregnancy test was performed for women who were not surgically sterile or were less than one year post-menopausal. A complete medical history was obtained. Height, weight, blood pressure, pulse, and temperature measurements were obtained.

Participants who met all eligible criteria were asked to refrain from citrus and citrus-containing productsfor a minimum of four weeks (washout period). Following the washout period, participants returned to the clinic for baseline NAF and plasma sample collection. NAF was collected into small capillary tubes and then immediately diluted in phosphate buffered saline (1:10). Plasma and diluted NAF were stored at $-80^{\circ} \mathrm{C}$ for limonene concentration determination. Following baseline sample collection, participants underwent their first massage session in the clinic. The massage oil was prepared fresh by blending 3 drops of organic orange oil (containing $0.14 \mathrm{~g}$ of limonene) with $1.35 \mathrm{~mL}$ base oil to give a final orange oil concentration of $10 \%$. Participants were instructed to wear surgical gloves and use their fingertips to massage the blended massage oil to the breasts in a circular fashion with slight pressure, avoiding areola and nipples, for 5 minutes. Participants were then provided with massage oil supplies for daily massage application. Participants were instructed that the massage application could be performed after showering or bathing and should be at least 8 hours before next showing/bathing. Participants were advised to avoid exposing their breasts to direct sunlight or a tanning bed throughout the intervention period and were provided with a daily diary to record adverse events and time of massage application. Following the 4-week daily massage intervention, participants 
returned to the clinic the day after their last massage application. Blood and NAF were collected and stored at $-80^{\circ} \mathrm{C}$ for limonene concentration determination. Additional blood samples were collected for post- intervention $\mathrm{CBC}$ and blood chemistry. Massage oil supplies and diary were examined to evaluate compliance. Adverse events were recorded for two more weeks after the massage intervention.

\subsection{Limonene Concentration Determination}

Limonene concentrations in plasma and NAFwere determined using a published assay [11] with minor modifications. Briefly, plasma or NAF samples $(50 \mu \mathrm{L})$ were mixed with an equal volume of the internal standard solution $(1.2 \mu \mathrm{g} / \mathrm{mL}$ of perillyl aldehyde in $100 \%$ acetonitrile) andthen extracted with hexane. An aliquot of the hexane layer was injected into the GC-MSsystem. The GC-MS system consisted of a GC with a PVT injector and a DSQ mass spectrometer (Thermo Electron Corporation, San Jose, CA, USA). Chromatographic separation of limonene and internal standard was achieved on a high resolution GC DB-5MS fused silica capillary column (AgilentTechnologies, Santa Clara, CA, USA). The calibration curve was linear over the concentration range of $5 \mathrm{ng} / \mathrm{mL}$ to $2000 \mathrm{ng} / \mathrm{mL}$.

Limonene concentrations in mouse mammary pads were analyzed according to a method developed in our laboratory with minor modifications [12]. Mammary pads were weighed and incubated at $37^{\circ} \mathrm{C}$ for 2.5 hours with $30 \mu \mathrm{L}$ of $30 \%$ potassium hydroxide and $100 \mu \mathrm{L}$ ethanol to induce saponification. After cooling to room temperature, $300 \mu \mathrm{L}$ hexane, $50 \mu \mathrm{L}$ purified $\mathrm{H}_{2} \mathrm{O}$, and 10 $\mu \mathrm{L}$ internal standard solution $(1.2 \mu \mathrm{g} / \mathrm{mL}$ of perillyl aldehyde in methanol) were added. Samples were vortexed and then centrifuged. An aliquot of the organic layer was injected into the GC-MS system as described above. Calibration curves were linear over the range of 6.25 2000 ng limonene.

\subsection{Statistical Analysis}

For the mouse study, plasma or mammary tissue limonene concentrations were compared between oral and topical administrationat the same dose levelusing the Wilcoxon rank sum test. Plasma or mammary tissue limonene concentrations were also compared between the two dose levels (10\% vs. $20 \%$ dose groups) for the same route of administrationusing the Wilcoxon rank sum test. For the clinical study, NAF or plasma limonene concentrations between baseline and post-intervention were compared using the signed rank test. A $P$-value of $<$ 0.05 was considered statistically significant for all analyses.

\section{Results}

\subsection{Mouse Disposition Study}

Table 1 summarizes mammary tissueand plasmalimonene concentrations from each treatment group. At $4 \mathrm{hrs}$ after the last dose, mammary tissue limonene concentrations were $47.95 \pm 33.96,44.55 \pm 13.21,46.00 \pm 14.37$, $115.4 \pm 85.43 \mu \mathrm{M}$ for topical application with $10 \%$ orange oil, oral administration with $10 \%$ orange oil, topical application with $20 \%$ orange oil, oral administration with $20 \%$ orange oil, respectively. All control groups had no detectable levels of limonene in the mammary tissue (data not shown). The limonene levels were not different between topical and oral administration. The limonene levels were also similar between $10 \%$ and $20 \%$ dose levels. Limonene levels in the mammary tissue were significantly higher than those in matched plasma; on average 1000-fold higher ( $P s<0.05)$.

There were no clinical signs of toxicity in any of the treatment groups. Mammary gland whole-mount preparations were evaluated for histopathological changes. As Figure 1 illustrates, no remarkable treatment-related changes in ductal structure or gland morphology were observed.

Table 1. Limonene levels in mouse mammary tissue and plasma after four weeks of daily oral or topical limonene administration.

\begin{tabular}{cccc}
\hline & $\begin{array}{c}\text { Mammary } \\
\text { tissue }(\mu \mathrm{M})\end{array}$ & Plasma $(\mu \mathrm{M})$ & $\begin{array}{c}\text { Mammary } \\
\text { tisue/Plasma ratio }\end{array}$ \\
\hline $\begin{array}{c}10 \% \text { Topical } \\
(\mathrm{N}=4)\end{array}$ & $47.95 \pm 33.96^{\mathrm{a}, \mathrm{b}}$ & $0.02 \pm 0.01$ & $4105 \pm 4183$ \\
$\begin{array}{c}20 \% \text { Topical } \\
(\mathrm{N}=4)\end{array}$ & $46.00 \pm 14.37^{\mathrm{b}}$ & $0.02 \pm 0.01$ & $3063 \pm 2286$ \\
$\begin{array}{c}10 \% \text { Oral } \\
(\mathrm{N}=4)\end{array}$ & $44.55 \pm 13.21^{\mathrm{b}}$ & $0.08 \pm 0.11$ & $1434 \pm 1195$ \\
$\begin{array}{c}20 \% \text { Oral } \\
(\mathrm{N}=4)\end{array}$ & $115.39 \pm 85.43^{\mathrm{b}}$ & $0.02 \pm 0.01$ & $4828 \pm 2516$ \\
\hline
\end{tabular}

${ }^{\mathrm{a}}$ Mean $\pm \mathrm{SD} ;{ }^{\mathrm{b}}$ Significantly higher than plasma $(P<0.05)$.

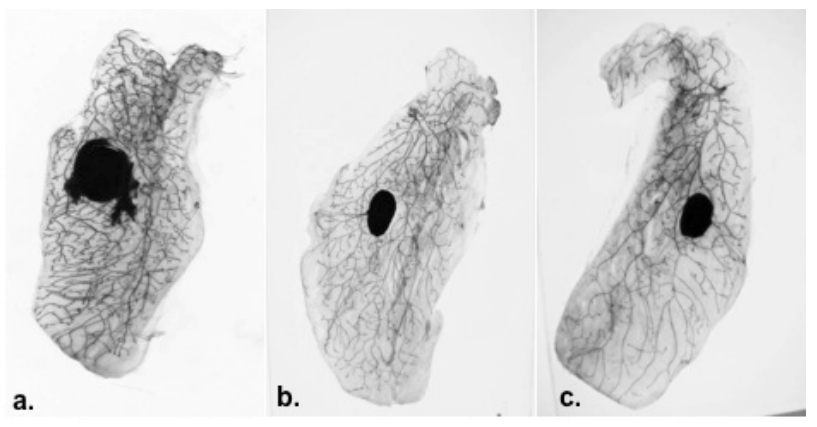

Figure 1. Mammary gland whole mounts (a) untreated control (b) topically applied limonene (c) orally administered limonene demonstrated that limonene did not adversely affect normal mammary gland morphology. 


\subsection{Human Safety and Tolerability Study}

Forty-four eligible women initiated the massage intervention. Table 2 summarizes participant demographic data. Four weeks of daily topical application of limonene containing massage oil was found to be well tolerated. One woman withdrew from the intervention after a single massage session due to intolerance to the citrus aroma. Forty-three completed the study intervention. Table $\mathbf{3}$ summarizes adverse events (AEs) deemed possibly or probably related to the study agent. Most AEs were mild and limited to dermatology-related events at the application site. Five women reported mild itching or tingling at the application site, but continued the study without dose-reduction. Four women developed a rash in the sun-exposed area of the application site. The rash was mild to moderate; all women completed the study without dose reduction. The orange oil preparation used in these women was analyzed by GC-MS and found to have increased levels of an oxygenated limonene by-product (limonene oxide), which has been previously associated with skin irritation [13]. Subsequent participants were

Table 2. Participants' demographic data.

\begin{tabular}{cc}
\hline Premenopausal Women & 16 \\
Age & 28 \\
Postmenopausal Women & $51.7 \pm 9.7^{\mathrm{a}}$ \\
Race: $n(\%)$ & $26.3 \pm 4.8^{\mathrm{a}}$ \\
Caucasian & \\
Black & $38(86.3)$ \\
Native American & $2(4.5)$ \\
Unknown & $2(4.5)$ \\
\hline${ }^{a}$ Mean + SD. & $2(4.5)$ \\
\hline
\end{tabular}

Table 3. Summary of possibly or probably related adverse events ${ }^{a}$.

\begin{tabular}{cc}
\hline Adverse Event & $n(\%)$ \\
\hline Itching & $5(11.4)$ \\
Rash & $4(9.1)$ \\
Dry Skin & $2(4.5)$ \\
Lightening Skin Color & $1(2.3)$ \\
Burning Sensation & $1(2.3)$ \\
Acne & $1(2.3)$ \\
Headaches & $1(2.3)$ \\
\hline
\end{tabular}

${ }^{\mathrm{a} A l l}$ events were mild grade except for one rash which was moderate grade. supplied with a new batch of orange oil that was tested to have undetectable levels of limonene oxide and no further participants experienced similar rashes. There were no changes in hematology measurements or blood chemistry following 4 weeks of orange oil application (data not shown). In addition, the intervention was found to have an average usage compliance of $96.8 \%$, calculated based on the number of massage oil vials used during the intervention.

Figure 2 illustrates baseline and post-intervention levels of limonene in matched NAF and plasmasamplesfrom 10 individuals who had sufficient NAF yield. Limonene levels in NAF were $95.11 \pm 50.02 \mathrm{nM}$ at baseline and did not change significantly after the intervention. Plasma limonene concentrations were $18.60 \pm 24.02 \mathrm{nM}$ at baseline and were also unchanged after the intervention. Interestingly, NAF limonene levels were significantly higher than the time-matched plasma $(P<0.01)$.

\section{Discussion}

The concept of topical application of drugs to the breast is novel and may provide potential for localized drug
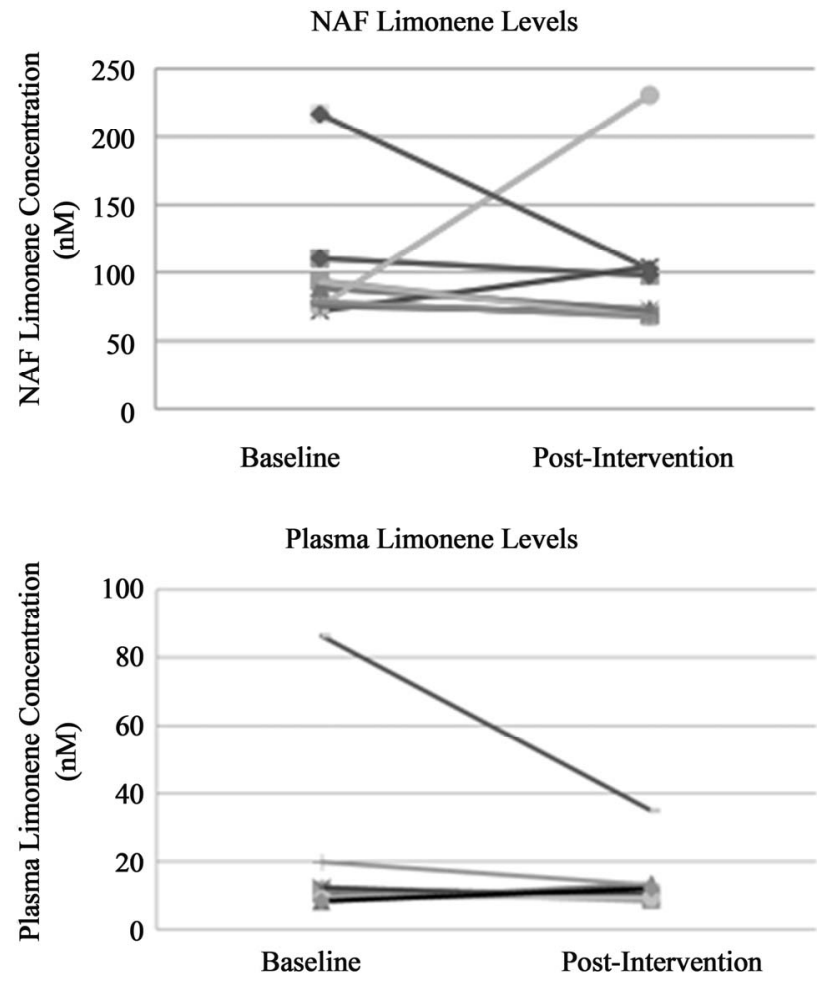

Figure 2. NAF and plasma limonene concentrations at baseline and after 4 weeks of topical application of limonene containing massage oil. Limonene levels in NAF were 95.11 $\pm 50.02 \mathrm{nM}$ at baseline and were not significantly different post-intervention. Plasma limonene concentrations were $18.60 \pm 24.02 \mathrm{nM}$ at baseline and were also not significantly different post-intervention. NAF limonene levels were significantly higher than the time-matched plasma $(P<0.01)$. 
delivery with minimal systemic side effects. Studies have shown that topical application of 4-hydroxyta- moxifen, the active metabolite of tamoxifen, to the breast resulted in effective mammary tissue concentration of 4-hydroxytamoxifen with much lower plasma levels [14]. A recent study showed that topical application of 4-hydroxytamoxifen to the breast resulted in reductions in breast tumor tissue proliferation indexes similar to that seen with oral administration of tamoxifen [15].

In our study, similar limonene levels were observed in the mouse mammary pads after oral and topical limonene administration as orange oil blended with base oil. Two different limonene dose levels (equivalent to 6 and $12 \mathrm{mg}$ limonene dose daily) did not result in a dose dependent increase in the limonene levels in the mammary pads. Consistent with its high lipophilicity and previous findings in humans [16] and rodents [17], limonene demonstrated preferential mammary tissue-to-plasma distribution in mice. Plasma concentrations of limonene were on average between $0.015-0.08 \mu \mathrm{M}$, with mammary tissue levels over 1000 -fold higher.

With the demonstration of mammary tissue bioavailability of limonene following topical application in mice, we conducted a pilot clinical study to determine the safety and feasibility of topical limonene application in healthy women. Our pilot study showed that topical application of limonene containing massage oil was met with excellent compliance and good safety profile. Side effects associated with orange oil application were mostly mild and limited to dermatological events at the application site. The rashes developed at the application site were most likely attributed to an oxidized limonene by-product and could be avoided by pre-testing the content of the oxidized limonene by-product in the orange oil supplies. Additionally, this study demonstrates that women are willing to apply a topical agent to their breast daily, providing a potential alternative for women unwilling or unable to take a daily oral drug.

Interestingly, limonene was detectable at baseline specimens in most participants despite of a 4-week washout period. Because limonene is present in many household cleaning products [18], in the environment [19], and in foods [20,21], presence of low levels of limonene in plasma and NAF at baseline was not unexpected. However, limonene levels were not changed in NAF or plasma after four weeks of daily orange oil application even though prior in vitro studies have shown that topically applied limonene can penetrate through human epidermis and dermis [8]. It is plausible that topically applied limonene as massage oil has limited absorption through human breast skin. It is also likely that dermally absorbed limonene, if any, may concentrate in the breast adipose tissue with minimal secretion into NAF or distribution into systemic circulation. Future clinical studies should consider incorporation of direct breast tissue sampling such as in women undergoing breast reduction surgery to assess tissue limonene levels.

In summary, topical administration of limonene to mice led to high mammary tissue limonene levels without affecting normal mammary gland morphology. A similar formulation given to women for topical application to the breast was met with high compliance and tolerability. Future research should assess the efficacy of topical limonene application in mammary cancer models and develop topical formulations that may further enhance breast tissue drug delivery.

\section{Acknowledgements}

We would like to thank Donna Vining and Heidi Fritz for their assistance in the clinical study conduct.

\section{REFERENCES}

[1] C. E. Elson, T. H. Maltzman, J. L. Boston, M. A. Tanner and M. N. Gould, "Anti-Carcinogenic Activity of dLimonene during the Initiation and Promotion/Progression Stages of DMBA-Induced Rat Mammary Carcinogenesis," Carcinogenesis, Vol. 9, No. 2, 1988, pp. 331332. doi:10.1093/carcin/9.2.331

[2] J. D. Haag, M. J. Lindstrom and M. N. Gould, "Limonene-Induced Regression of Mammary Carcinomas," Cancer Research, Vol. 52, No. 14, 1992, pp. 4021-4026.

[3] T. H. Maltzman, L. M. Hurt, C. E. Elson, M. A. Tanner and M. N. Gould, "The Prevention of NitrosomethylureaInduced Mammary Tumors by d-Limonene and Orange Oil," Carcinogenesis, Vol. 10, No. 4, 1989, pp. 781-783. doi:10.1093/carcin/10.4.781

[4] M. N. Gould, C. J. Moore, R. Zhang, B. Wang, W. S. Kennan and J. D. Haag, "Limonene Chemoprevention of Mammary Carcinoma Induction following Direct in Situ Transfer of V-Ha-Ras," Cancer Research, Vol. 54, No. 13, 1994, pp. 3540-3543.

[5] J. A. Elegbede, C. E. Elson, M. A. Tanner, A. Qureshi and M. N. Gould, "Regression of Rat Primary Mammary Tumors following Dietary d-Limonene," Journal of the National Cancer Institute, Vol. 76, No. 2, 1986, pp. 323325.

[6] K. Zhao and J. Singh, "Mechanism(s) of in Vitro Percutaneous Absorption Enhancement of Tamoxifen by Enhancers," Journal of Pharmaceutical Sciences, Vol. 89, No. 6, 2000, pp. 771-780.

doi:10.1002/(SICI)1520-6017(200006)89:6<771::AID-JP S9>3.0.CO;2-Y

[7] P. A. Cornwell, B. W. Barry, C. P. Stoddart and J. A. Bouwstra, "Wide-Angle X-Ray Diffraction of Human Stratum Corneum: Effects of Hydration and Terpene Enhancer Treatment," Journal of Pharmacy and Pharmacology, Vol. 46, No. 12, 1994, pp. 938-950. doi:10.1111/j.2042-7158.1994.tb03248.x

[8] K. Cal, S. Janicki and M. Sznitowska, "In Vitro Studies 
on Penetration of Terpenes from Matrix-Type Transdermal Systems through Human Skin," International Journal of Pharmaceutics, Vol. 224, No. 1-2, 2001, pp. 81-88. doi:10.1016/S0378-5173(01)00744-X

[9] J. R. Trevithick and K. P. Mitton, "Uptake of Vitamin E Succinate by the Skin, Conversion to Free Vitamin E, and Transport to Internal Organs," Biochemistry \& Molecular Biology International, Vol. 47, No. 3, 1999, pp. 509-518.

[10] J. A. Schroeder, A. A. Masri, M. C. Adriance, J. C. Tessier, K. L. Kotlarczyk, M. C. Thompson and S. J. Gendler, "MUC1 Overexpression Results in Mammary Gland Tumorigenesis and Prolonged Alveolar Differentiation," Oncogene, Vol. 23, No. 34, 2004, pp. 5739-5747. doi:10.1038/sj.onc. 1207713

[11] S. Wang, Y. Chen, Z. Gao, M. Xiong, Z. Zhong and L. Ye, "Gas Chromatographic-Mass Spectrometric Analysis of d-Limonene in Human Plasma," Journal of Pharmaceutical and Biomedical Analysis, Vol. 44, No. 5, 2007, pp. 1095-1099. doi:10.1016/j.jpba.2007.04.018

[12] J. A. Miller, I. A. Hakim, C. Thomson, P. Thompson and H. H. Chow, "Determination of d-Limonene in Adipose Tissue by Gas Chromatography-Mass Spectrometry," Journal of Chromatography B-Analytical Technologies in the Biomedical and Life Sciences, Vol. 870, No. 1, 2008, pp. 68-73. doi:10.1016/j.jchromb.2008.06.002

[13] M. Matura, A. Goossens, O. Bordalo, B. Garcia-Bravo, K. Magnusson, K. Wrangsjo and A. T. Karlberg, "Patch Testing with Oxidized R-(+)-Limonene and Its Hydroperoxide Fraction," Contact Dermatitis, Vol. 49, No. 1, 2003, pp. 15-21. doi:10.1111/j.0105-1873.2003.00135.x

[14] H. Pujol, J. Girault, P. Rouanet, S. Fournier, J. Grenier, J. Simony, J. B. Fourtillan and J. L. Pujol, "Phase I Study of Percutaneous 4-Hydroxy-Tamoxifen with Analyses of 4Hydroxy-Tamoxifen Concentrations in Breast Cancer and Normal Breast Tissue," Cancer Chemotherapy and Pharmacology, Vol. 36, No. 6, 1995, pp. 493-498.

\author{
doi:10.1007/BF00685799
}

[15] P. Rouanet, G. Linares-Cruz, F. Dravet, S. Poujol, S. Gourgou, J. Simony-Lafontaine, J. Grenier, A. Kramar, J. Girault, E. Le Nestour, et al., "Neoadjuvant Percutaneous 4-Hydroxytamoxifen Decreases Breast Tumoral Cell Proliferation: A Prospective Controlled Randomized Study Comparing Three Doses Of 4-Hydroxytamoxifen Gel to Oral Tamoxifen," Journal of Clinical Oncology, Vol. 23, No. 13, 2005, pp. 2980-2987. doi:10.1200/JCO.2005.06.064

[16] J. A. Miller, I. A. Hakim, W. M. Chew, P. A. Thompson, C. A. Thomson and H. H. Chow, "Adipose Tissue Accumulation of D-Limonene with the Consumption of a Lemonade Preparation Rich in D-Limonene Content." Nutrition and Cancer, Vol. 6, No. 62, 2010, pp. 783-788. doi:10.1080/01635581003693066

[17] P. L. Crowell, S. Lin, E. Vedejs and M. N. Gould, "Identification of Metabolites of the Antitumor Agent dLimonene Capable of Inhibiting Protein Isoprenylation and Cell Growth," Cancer Chemotherapy and Pharmacology, Vol. 31, No. 3, 1992, pp. 205-212. doi:10.1007/BF00685549

[18] M. Magnano, S. Silvani, C. Vincenzi, M. Nino and A. Tosti, "Contact Allergens and Irritants in Household Washing and Cleaning Products," Contact Dermatitis, Vol. 61, No. 6, 2009, pp. 337-341. doi:10.1111/j.1600-0536.2009.01647.x

[19] Association FaEM, “d-Limonene Monograph,” No. 1991, pp. 1-4.

[20] Geneva WHO, International Programme on Chemical Safety (No. 0918), "International Chemical Safety Cardd-Limonene," 1993.

[21] A. S. Malik, O. Boyko, N. Atkar and W. F. Young, "A Comparative Study of MR Imaging Profile of Titanium Pedicle Screws," Acta Radiologica, Vol. 42, No. 3, 2001, pp. 291-293. doi:10.1080/028418501127346846 